\section{Normas para el desarrollo de programas de educación sobre la diabetes en América}

\author{
Comité de Educación, DOTA
}

Palabras clave: diabetes, educación, diabetológica, DOTA.

\footnotetext{
1 Comite de Educación, DOTA (Declaration of the Americas): Pablo Aschner, Presidente, Asociación Latinoamericana de Diabetes (ALAD); Alberto Barceló, Asesor Regional, Organización Panamericana de la Salud (OPS); Charles M. Clark Jr., Presidente, Programa Nacional de Educación en Diabetes de los Estados Unidos de América (National Diabetes Education Program: NDEP); Tony Elphick, Representante en DOTA, Novo Nordisk A/S; Juan Jose Gagliardino, Director, Centro de Endocrinología Experimental y Aplicada (CENEXA), Centro Colaborador de la Organización Mundial de la Salud (OMS)/OPS; Christine Karkashian, Consultora sobre Educación en Diabetes, OPS; Beth McBride, Presidente, Grupo de Educación de la Federación Internacional de Diabetes-Norteamérica (North America-International Diabetes Federation: NA-IDF); Kathy Mulcahy, Relaciones Internacionales, Asociación Americana de Educadores en Diabetes (American Association of Diabetes Educators: AADE); Adolfo Pérez Comas, Presidente, Comité de Educación de la DOTA y Presidente, Federación Internacional de Diabetes-Sudamérica y Centroamérica (South and Central America-International Diabetes Federation: SACA-IDF); Betsy Rodriguez, Copresidente, Grupo de Educación de SACA-IDF; Rita Saltiel-Berzin, Gerente de Educación, Becton Dickinson \& Co. Toda la correspondencia debe ser enviada al Grupo de Educación, a la siguiente dirección: DOTA Office, 1701 N Beauregard St., Alexandria, VA 22311, Estados Unidos de América. Correo electrónico: DOTA@diabetes.org
}

La Declaración de las Américas (Declaration of the Americas: DOTA) es un reconocimiento de la gravedad de la diabetes en el continente y un compromiso de los gobiernos de la Región para poner en práctica estrategias y acciones capaces de reducir el costo socioeconómico de la enfermedad y mejorar la calidad de vida de quienes la padecen. En ese contexto se inscribe la necesidad de incorporar la educación diabetológica como una prestación médica indispensable para lograr la participación activa del paciente en el control y tratamiento de la enfermedad.

La promoción de la educación del paciente como premisa fundamental para el tratamiento de la diabetes ya la hacía Bouchardat en 1875, y su valor ha sido demostrado reiteradamente por diversos autores en comunidades de muy distintas características socioeconómicas. Sin embargo, solo un reducido número de pacientes de algunos países recibe una adecuada educación diabetológica. Por consiguiente, los pacientes ignoran aspectos fundamentales de su enfermedad y los pasos a seguir para controlarla. Esta situación es debida, en parte, a que la educación no tiene aún el suficiente prestigio científico y sus resultados no son bien conocidos por los líderes de opinión y por los responsables de la salud pública.

Para ser eficaz, la educación requiere una serie de condiciones tales como formación, conocimientos y aptitudes pedagógicas, buena capacidad de comunicación y capacidad para escuchar, comprender y negociar. Para asegurar un impacto positivo de la educación es necesario que quienes la ejercen tengan formación y experiencia adecuadas y que sea reconocida como una prestación esencial $\mathrm{y}$, por lo tanto, se remunere de modo satisfactorio.

Para facilitar el logro de estos objetivos, el Comité de Educación de la DOTA elaboró el presente documento en el cual se enumeran las condiciones que debe reunir un programa de educación de pacientes con diabetes. Para esto se analizaron otros documentos referidos al tema, como los de la Federación Internacional de Diabetes (International Federation of Diabetes: IDF), la Asociación Americana de Diabetes (American Diabetes Association: ADA) y la Asociación Americana de Educadores en Diabetes (American Association of Diabetes Educators: AADE). Por consiguiente, muchos de los conceptos vertidos en el presente documento coinciden con los incluidos en los documentos consultados. 
La Región de las Américas es heterogénea desde el punto de vista étnico, cultural, de las tradiciones, del grado de alfabetización, de los sistemas de salud y de las condiciones socioeconómicas. Esta heterogeneidad también se refleja en el grado de difusión, importancia concedida, implementación y eficacia de los programas de educación en diabetes. En consecuencia, la tarea de establecer pautas no es sencilla, pues se corre el riesgo simultáneo de fijar objetivos inferiores a los ya vigentes para algunos, y metas que parecen inalcanzables para otros. En estas circunstancias, el Comité de Educación de la DOTA trató de uniformar criterios y establecer normas comunes que permitan a los diabéticos de la Región acceder a una educación diabetológica razonable que los incorpore activa y efectivamente al control y tratamiento de su enfermedad.

El Comité de Educación desearía que las normas enumeradas no solo se cumplieran, sino que se superaran en los programas educativos de la Región. Sin embargo, consciente de la situación de muchos países en los que estos programas están todavía en fase embrionaria y para evitar la inacción, ha definido en un apéndice a este documento los criterios mínimos a alcanzar para considerar eficaz un programa. Consideramos que el contenido del documento facilitará la tarea tanto de los responsables de la educación de los pacientes como de quienes tienen el poder de acreditación de los programas educativos como parte del tratamiento de esta enfermedad.

Por lo expuesto, por la necesidad de evaluar sus resultados y por la permanente ampliación de conocimientos en el campo de la diabetes, el Comité de Educación estima que este documento necesitará actualizaciones periódicas para asegurar su vigencia y eficacia. Ese es y será su compromiso y desafío.

La aplicación de estas normas ofrece a las personas con diabetes la oportunidad de prevenir la aparición de las complicaciones crónicas de la enfermedad y mejorar su calidad de vida. Aunque somos conscientes de que esta no es una tarea fácil, creemos que los beneficios obtenibles justican el esfuerzo.

\section{NORMAS}

\section{Organización}

Se creará una organización responsable del diseño y puesta en práctica del programa de educación en diabetes, integrada por personal cualificado y compuesta por un Coordinador y un Comité Asesor.

- Norma 1. La organización definirá claramente y enunciará por escrito los objetivos del programa, estableciendo que la educación es un componente fundamental del tratamiento de la diabetes.

- Norma 2. La organización proporcionará los recursos necesarios para lograr dichos objetivos, teniendo en cuenta la población de interés y sus carcterísticas socioculturales. Estos recursos deben ser suficientes en lo referente a espacio/local, personal, presupuesto y materiales educativos (audiovisuales, manuales para los participantes, láminas con información educativa y otros).

- Norma 3. La estructura docente-administrativa incluirá los siguientes componentes: el Equipo y sus integrantes, el Coordinador y el Comité Asesor.

\section{Características del personal de la organización}

- Norma 4. El personal de la organización cumplirá los siguientes requisitos:

a. Debe tener conocimientos sobre la diabetes - en sus distintos aspectos, tales como diagnóstico, control y tratamiento- y los principios educativos (aspectos pedagógicos, motivacionales, evaluativos).

b. El equipo estará formado, como mínimo, por un médico y un educador en diabetes. Este último puede ser un profesional de la salud $u$ otro profesional que acredite formación específica y suficiente educación diabetológica, certificadas por una autoridad competente.

c. También es deseable la incorporación de otros miembros del equipo de salud, como nutricionistas, podólogos, profesores de educación física, psicólogos y trabajadores sociales.

d. El equipo se reunirá con el coordinador, como mínimo tres veces al año, para intercambiar opiniones, evaluar la marcha del programa y elevar un informe al Comité Asesor.

\section{El coordinador}

- Norma 5. El coordinador será responsable de la planificación, funcionamiento y evaluación del programa. Además:

a. Actuará como enlace entre el equipo y el Comité Asesor.

b. Proporcionará y coordinará la educación continua y progresiva del equipo y del personal de la organización.

c. Se reunirá periódicamente con el equipo, como mínimo tres veces al año.

d. Participará en la planificación y revisión anual del programa. 
e. Participará en la preparación del presupuesto.

f. Será miembro del Comité Asesor.

\section{Comité Asesor}

- Norma 6. El Comité Asesor tendrá las siguien-tes características y modalidades de funcionamiento:

a. Tendrá una composición interdisciplinaria e intersectorial, con representación del equipo de salud, los pacientes y la comunidad. El representante comunitario no tendrá relación de dependencia con la organización.

b. Todos sus miembros tendrán experiencia en la caracterización y el control de la diabetes.

c. Se reunirá al menos dos veces al año.

d. Participará anualmente en la planificación del programa: objetivos, accesibilidad, métodos de enseñanza, recursos, seguimiento $\mathrm{y}$ evaluación.

e. Revisará y aprobará anualmente los programas y recomendaciones.

f. Aprobará nuevos programas que se ajusten a los criterios preestablecidos.

g. Certificará la idoneidad (conocimientos, aptitudes y experiencia) de los educadores.

\section{Programa de Educación}

- Norma 7. Al desarrollar el programa se procurará que sea accesible a toda la población a la que va dirigido, considerando los diferentes tipos de diabetes (tipo 1, tipo 2 y diabetes gestacional), las diferentes edades y las necesidades especiales de algunos grupos específicos de pacientes.

- Norma 8. Se garantizará que el programa se desarrolle de forma regular y sistemática, y asegure la educación continuada de sus participantes (educadores y educandos).

\section{Población}

- Norma 9. Se definirá la población a educar, teniendo en cuenta el número potencial de pacientes, el tipo de diabetes, la edad, el idioma, las características regionales y las necesidades educativas especiales (por ejemplo, grado de escolaridad y de analfabetismo).

- Norma 10. Se elaborará un documento que estipule claramente el currículo del programa educativo y refleje sus objetivos y contenidos, la metodología a emplear, los materiales educativos que se utilizarán y la forma de evaluar sus logros (instrumentos de evaluación, frecuencia de la misma y responsable de su realización).

\section{Currículo del programa}

- Norma 11. El programa educativo incluirá los siguientes aspectos:

a. Generalidades sobre diabetes.

b. Factores psicosociales y estrés.

c. Apoyo social y participación familiar.

d. Nutrición.

e. Actividad física.

f. Medicación especifica de la DM y de los factores de riesgo asociados.

g. Monitorización clínica y metabólica por el propio enfermo, su realización, interpretación de sus resultados y decisiones que la acompañan.

h. Relación entre alimentación, actividad física, medicación y glucemia y otros parámetros metabólicos.

i. Prevención, detección y tratamiento de las complicaciones agudas y crónicas.

j. Cuidados dentales y cutáneos, con especial énfasis en el cuidado de los pies.

k. Beneficios, riesgos y empleo de diferentes alternativas para lograr un mejor control metabólico variables clínicas, metabólicas y terapéuticas y de los factores de riesgo cardiovascular.

1. Cuidados en la etapa preconceptiva, durante el embarazo y en la diabetes gestacional.

m. Utilización de los sistemas de salud y recursos de la comunidad.

n. Indicaciones para situaciones especiales, como las enfermedades intercurrentes o los viajes.

o. Ambiente laboral.

p. Consecuencias negativas de hábitos como el tabaquismo o el consumo de alcohol y formas de eliminar estas conductas.

q. Estrategias para cambiar de conducta, establecer y cumplir los objetivos personales con respecto al tratamiento médico, reducir los factores de riesgo y superar los conflictos en la vida diaria.

- Norma 12. El programa educativo utilizará métodos y materiales apropiados según las características del grupo a educar.

\section{Metodología de Enseñanza}

El programa debe incluir planes educativos individuales y grupales.

- Norma 13. La enseñanza individual será una responsabilidad compartida con el grupo encargado de la atención al paciente y se hará en cada con- 
sulta, de manera que cada acto médico sea un acto educativo. El equipo acordará con el paciente las posibles modificaciones en el desarrollo del programa, de acuerdo con sus necesidades individuales, basadas en los resultados de evaluaciones periódicas.

- Norma 14. La enseñanza grupal no es un sustituto de la individual, sino su complemento, en particular de la recibida directamente durante las consultas regulares con los otros integrantes del equipo de salud. Se promoverá la formación de grupos pequeños (máximo de 15 personas) y se utilizarán sobre todo técnicas participativas que faciliten la permanente comunicación entre educando y educador. Se promoverá la toma de decisiones del paciente con respecto a adaptaciones y cambios de hábitos, control y tratamiento que potencien las acciones del equipo de salud en beneficio de su grado de control, la prevención de complicaciones y el mejoramiento de su calidad de vida. Se procurará formar grupos tan homogéneos como sea posible, teniendo en cuenta la edad de los educandos, el tipo de diabetes, el nivel sociocultural de los participantes y la existencia de potenciales barreras al aprendizaje.

\section{Evaluación}

Los resultados del programa educativo serán revisados anualmente por el coordinador y el Comité Asesor. Del resultado de esta evaluación dependerán sus modificaciones futuras. Se determinará si el programa sigue cumpliendo las normas establecidas. Los resultados deben ser documentados y utilizados en la subsecuente planificación y actualización del programa. De no cumplirse la evaluación en tiempo y forma o de no alcanzarse los objetivos preestablecidos, el Comité Asesor estará autorizado a intervenir e incluso a suspender el funcionamiento de los equipos.

- Norma 15. El Comité Asesor debe realizar una evaluación anual del programa educativo, teniendo en cuenta los siguientes aspectos:

a. Objetivos.

b. Currículo, métodos y materiales.

c. Composición del equipo.

d. Accesibilidad del programa y mecanismos de seguimiento de los participantes.

e. Recursos del programa (espacio, personal y presupuesto).

f. Estrategias de mercadotecnia para lograr un mayor acceso a la población de interés.

g. Eficacia del programa basado en datos objetivos (clínicos, bioquímicos, terapéuticos, económicos y de satisfacción) de los pacientes que asisten al programa. Los participantes se evaluarán al inicio del programa, inmediatamente después de su conclusión y, por lo menos, 6 y 12 meses más tarde.

La información obtenida se utilizará para evaluar la eficacia del programa, identificar los aspectos que necesiten ser reforzados o modificados, realizar adaptaciones en función del cambio de demanda de los participantes e incluir temas de interés en cursos de educación continua.

- Norma 16. El conocimiento adquirido sobre la diabetes debe evaluarse de acuerdo con los objetivos del programa, incluyendo como mínimo los siguientes parámetros:

a. Cambios clínicos: peso, síntomas, hipoglucemia, cetoacidosis, internamientos, cambios de medicación, tensión arterial.

b. Cambios de laboratorio: glucemia, glucosuria, cetonuria, $\mathrm{HbA}_{1 c^{\prime}}$, perfil lipídico.

c. Actitudes: automonitoreo, adherencia al plan de alimentación, actividad física.

d. Utilización de servicios.

e. Aspectos psicosociales, tales como creencias sobre la salud, grado de apoyo familiar, nivel socioeconómico, satisfacción y barreras al aprendizaje.

\section{APÉNDICE}

Las 16 normas descritas en este documento deben ser seguidas por todos los programas de educación en diabetes que esperen alcanzar un nivel de excelencia, especialmente, si incluyen no solo a pacientes, sino también a personas que van a ser entrenadas como educadores. Sin embargo, esto último requiere un alto grado de desarrollo estructural, además de recursos económicos y humanos.

Un programa educativo que cumpla con todas las normas propuestas puede ser puesto en práctica en Centros de Diabetes, tal como los define la Organización Mundial de la Salud (OMS) (1). Los Centros de Diabetes ofrecen una amplia gama de programas de asistencia sanitaria multidisciplinaria y deben disponer, al menos, de un diabetólogoendocrinólogo, dos profesionales de educación , varios especialistas médicos que aseguren el diagnóstico temprano, la prevención y el tratamiento de las complicaciones de la diabetes, y otros profesionales de la salud que puedan aportar conocimientos complementarios. Desdichadamente, la posibilidad de establecer Centros de Diabetes en América Latina y el Caribe es limitada. Estos centros pueden servir como centros terciarios de servicios asistenciales y 
pueden estar conectados con equipos o unidades de atención primaria.

En el ámbito de la atención primaria, los servicios deben ser prestados por médicos que sean capaces de efectuar diagnósticos básicos, prescribir medicamentos esenciales y organizar programas educativos simples descritos en las normas $4 a, 7,8$, $9,10,11,12,14$ y 16. Lo óptimo sería contar con un equipo compuesto por un médico con interés y experiencia en el control y tratamiento de la diabetes y un profesional de la educación. Este equipo podría consituir la organización, con el médico actuando como coordinador que cumpliría con las normas 1, 2, 4b, 5 y 13 .

En el ámbito de la atención secundaria, lo mínimo deseable sería un Equipo de Diabetes, aunque lo óptimo es una Unidad de Diabetes. El equipo estaría compuesto por un diabetólgo-endocrinólogo, un internista especializado en diabetes, un profesional de la educación $\mathrm{y}$, por lo menos, tres profesionales de la salud que presten asistencia multidisciplinaria. Este personal puede implementar el programa educativo, aunque el Comité Asesor podrá encontrarse con que es difícil cumplir los requisitos 15d, e, f, y g, a menos que la Unidad se amplíe y se convierta en un centro.

\section{SYNOPSIS}

\section{Standards for developing diabetes education programs in the Americas}

The Declaration of the Americas (DOTA) recognizes the severity of diabetes in the Region of the Americas and the commitment of the governments of the Region to implement strategies and actions that can reduce the socioeconomic cost of diabetes and improve the quality of life of those who suffer from the disease. Given that, there is a need to incorporate diabetes education as an indispensable medical service in order to achieve the active participation of patients in controlling and treating the disease. The DOTA Education Committee prepared this document to specify the conditions and standards that a patient diabetes-education program should meet.

\section{REFERENCIAS}

1. Reiber GE, King H. Guidelines for the development of a National Programme for Diabetes Mellitus. WHO/DBO/DM/91.1.
2. American Diabetes Association. National standards for diabetes self-management education. Diabetes Care 1999;22:S111S114.
3. International Diabetes Federation. International consensus position statements for diabetes education. London: IDF Consultative Section on Diabetes Education; 2000.

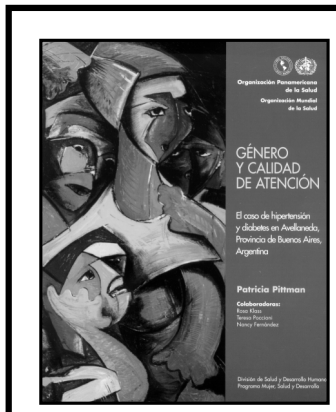

$1999 \cdot 128 \mathrm{pp}$ ISBN 9275323070 Código: OT 119

Precios:US\$20.00/

US\$ 16.00 en América Latina y el Caribe

\section{Género y calidad de atención : El caso de hipertensión y diabetes en Avellaneda, Provincia de Buenos Aires, Argentina}

Interesante estudio que forma parte de los esfuerzos de la OPS/OMS a fin de que se reconozca en todo el orbe la necesidad de integrar un enfoque de género a las políticas, programas y proyectos de salud. El objetivo fue detectar la posible existencia de problemas específicos de género en la atención a personas con hipertensión y diabetes tipo II, en una zona de pobreza extrema, en Buenos Aires. Participaron 28 sujetos, de los cuales la mitad eran mujeres. Los hallazgos, a pesar de ser locales, aportan elementos básicos para valorar las diversas formas en que las relaciones de género inciden en el proceso de salud-enfermedad-atención. Una versión simplificada del protocolo se aplicó ya en Nicaragua, El Salvador, Guatemala y Honduras.

Ciertamente la presente publicación será de suma utilidad para mejorar la calidad de la atención primaria a la salud que brindan médicos, enfermeros y administrativos, particularmente en zonas marginadas de los países en vías de desarrollo.

http://publications.paho.org • Fax: (301) 206-9789・Correo electrónico:paho@pmds.com 